\title{
Influence of Temperature Phase Grating Relaxation Times on the Lasing Characteristics of Q-Switched and Mode-Locked Nd:YAG Laser-Pumped Distributed Feedback Dye Lasers
}

\author{
Nasrullah KHAN and Norman MARIUN \\ Department of Electrical and Electronic Engineering Faculty of Engineering, \\ University Putra Malaysia 43400 UPM Serdang, Selangor, Malaysia
}

(Received March 11, 2003)

\begin{abstract}
This work presents a theoretical explanation and experimental evidence of pulses- intensity displacement and pulse- broadening profiles of a Q-switched and mode-locked Nd:YAG laser- pumped distributed feedback dye laser (DFDL). Experimental results followed by a theoretical simulation indicate that DFDL pulses peaked noticeably later than their corresponding Nd:YAG laser pulses. The DFDL pulse corresponding to the peakpower pump laser pulse occurs few pulses later than the expected location. The observed and simulated delays were found to depend upon the inter-pulse periods of the pumping Q-switched and mode-locked laser. We report the theoretical basis for the influence of temperature grating relaxation times on the dye laser's pulse characteristics, and apply the transient grating technique to measure the refractive index of a lasing dye solution. Results indicate that the thermal effects of preceding pulses integrate the gain for receding pulses. These results provide a good understanding of temperature grating relaxation times and their impact on the lasing characteristics of DFDL.
\end{abstract}

Key Words: Bragg grating, Thermal phase grating, Temperature grating, Gain grating and DFDL

\section{Introduction}

Peak pulse shifting phenomenon was first time reported in context of laser over exposure analysis study by the author elsewhere $^{1)}$ and similar observations such as pulse and spectral broadening phenomenon were noted by several other others. ${ }^{2,3)}$ Kurstak and Aufrick, Belarus, have reported that thermal phase lattice in nanosecond pulse excited distributed feedback dye lasers can strongly influence the lasing characteristics such as pulse durations. The thermal gratings influence the feedback characteristics that consequently change the duration of output pulses. In a Q-switched and mode-locked pumped distributed feedback dye laser the last pulse may be 1-2 times longer than the first pulse. ${ }^{2)}$ Furthermore, they reported that temperature phase grating effects can be minimized by reducing the pump laser energy that is consistent with present findings. However, in this study it will be illustrated that the duration elongation also depends upon dye concentration, modal period, number of pump pulses and their intensities.

J. Liang and $\mathrm{H}$. Sun et $\mathrm{al}^{3)}$ have observed lasing wavelength shift from reflection center of a doped fiber-grating laser. The peak lasing pulse occurs little bit later than expected. They supposed that this phenomenon does not depend on the temperature or reflection of Bragg grating rather it depends upon some kind of gain mechanism or re-absorption within the lasing medium. Several others have reported similar observations in different disciplines of science and engineering. ${ }^{4-8)}$ Boerman et $\mathrm{al}^{4)}$ found that thermal diffusivity in elastomer increases linearly to a value of some $10 \%$ lower than the equilibrium value using forced Rayleigh scattering technique. In fact temperature takes few hundred ps to several ns to relax, depending upon the nature of solution and its additives. This study is focused on theoretical and experimental investigation of various parameters and lasing characteristics of distributed feedback dye lasers. The influence of the gradual accumulation of the amplitude of temperature gratings in a dye cell has been studied with a passively Q-switched and mode locked Nd:YAG laser. It will be shown that the peak of the pulse envelopes of the Nd:YAG laser and DFDL do not corresponding time; rather the peak of the DFDL envelope is delayed from the peak of the pump laser envelope. The delay between the peaks of the envelopes depends upon the inter-pulse period $2 L_{\mathrm{c}} / c$ of the pump laser. A computer simulation program using Fortran 77 calculated the observed increase in temperature grating amplitudes.

\section{Experimental setup}

Second harmonic of a laboratory built passively Q-switched and mode-locked Nd:YAG laser was used to excite the solution of Rh6G in ethanol at concentration of $1.0 \times 10^{-3} \mathrm{M}$. The experimental arrangement is shown in Fig. 1. The concentration of mode-locking dye (Eastman Kodak \#9740) in the solvent (Cholorobenze) was adjusted at a constant pump power so as to produce nearly six pulses whose intensity was above the half maximum. Second harmonic was generated by a BBO crystal. The unconverted IR was reflected at $45^{\circ}$ for $1.06 \mu \mathrm{m}$. The un- 


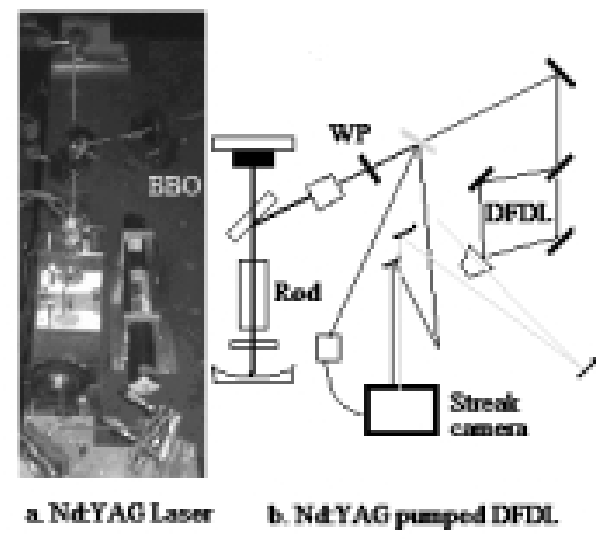

Fig. 1 Experimental setup for Nd:YAG laser pumped DFDL.

converted IR was used to trigger the Hadland Imacon streak camera. The $5 \%$ green energy reflected along with the IR was used as reference signal. A dielectric beam splitter was used to split the forward beam to cause DFDL operation at dye cell. A cylindrical telescope was inserted just before the beam splitter to focus the pump light onto the dye cell in a focal line of 2-3 mm length. Both lines were superimposed upon each other. The reference green signal and the DFDL output $(569 \mathrm{~nm})$ light were made to traverse equal optical paths lengths of 8 meters before entering the streak camera entrance slit. The emphasis was laid on equalizing the optical path differences between the two signals.

The green and yellowish signals were adjusted by suitable combinations of spherical and cylindrical lenses to appear in the center of the streak camera intensifier screen. Anumber of streak records were taken on Polaroid-47 films for initial adjustment and on HP-5 film for final observations. The streak records were taken at a sweep speed of 1-2 ns/mm so that maximum number of pulses could be recorded. The streak camera calibration was done using known inter-pulse periods $\left(2 L_{\mathrm{c}} / c\right)$. The apparent sweep speed of 1 and $2 \mathrm{~ns} / \mathrm{mm}$ were recalibrated to be equal to $2.87 \pm 0.15 \mathrm{~ns} / \mathrm{mm}$ and $5.68 \pm 0.1 \mathrm{~ns} / \mathrm{mm}$ respectively. The experiments were repeated for three different values of $2 L_{\mathrm{c}} / c$. The inter-pulse periods were varied by changing the cavity length of the Nd:YAG laser.

\section{Description of results}

The streak records taken on HP-5 film were exposed with light through a step filter array before processing. The negatives were scanned by a computer-controlled microdensitometer. The step filter array negatives ware scanned in steps of $2 \mathrm{~mm}$ and the lasers streak records were scanned at step interval of $0.05 \mathrm{~mm}$. The scanned data was plotted using MS Excel. Variation of optical density as function of $\log I$ am shown in Fig. 2. The data of optical density and Log $I$ was plotted on a Macintosh computer to determine an approximate polynomial equation using curve fitting program. The polynomial equation for intensity in terms of optical density was found to be

$$
\log I=1.377-1.0083 \rho+0.3363 \rho^{2}-0.0436 \rho^{3}+0.0022 \rho^{4}
$$

where $\rho$ is the optical density of film. The data of laser streak records for optical density were converted into optical intensity using polynomial equation. The converted intensity as a function of scanning rate was plotted using an Excel spread sheet. A

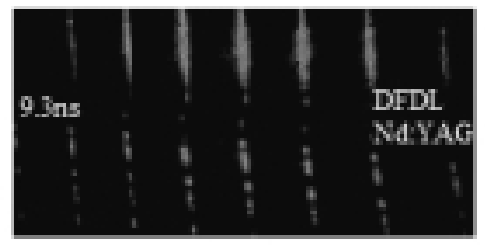

a. Streak camera records of pulses

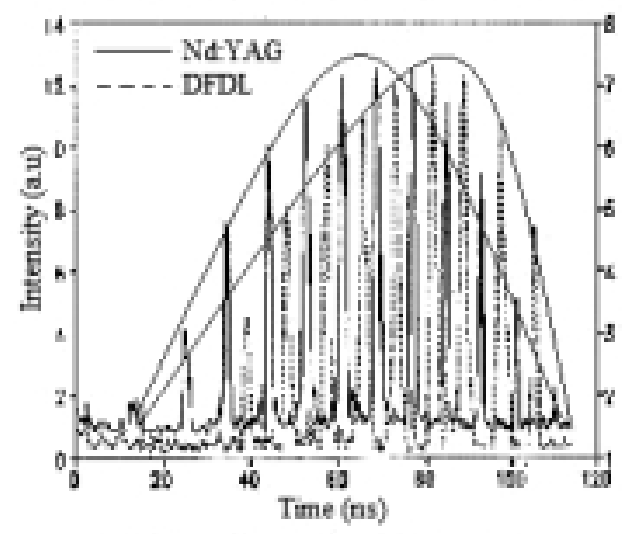

b. Microdensitometer scanned plots

Fig. 2 Microdensitometer scanned streak record of Qswitched and mode locked Nd:YAG laser and corresponding DFDL output for inter-pulse period of 8.31 ns.

typical trace of a streak record for inter-pulse period of $8.31 \mathrm{~ns}$ of the Nd:YAG laser with its plot is shown in Fig. 2. The peaks of the Nd:YAG laser pulse envelope (dotted) and the DFDL pulse envelope (solid) are clearly delayed from each other.

\section{Model for thermal gain accumulation}

When a dye cell is pumped by the interference patterns of a mode locked laser a temperature grating is induced within the dye solution and this takes a certain time to relax to the initial temperature. If there are more than one excitation pulses then the temperature effects of previous pulses contribute to the grating. The phenomenon becomes very important when the inter-pulse interval $2 L_{\mathrm{c}} / c$ is very short. The thermal heat transfer to the subsequent pulses depend entirely on the comparison of the thermal constant $\tau$ and the inter-pulse period $2 L_{\mathrm{c}} / c$, which in turn is determined by the cavity length of the passively Qswitched and mode locked pump laser. To investigate it I have developed a model, which takes into account the thermal relaxation of temperature gratings. The postulations on this model for an actively mode locked laser were reported elsewhere ${ }^{10)}$

\section{Model for Q-switched and mode-locked excitation laser}

If the dye cell is pumped by a passively Q-switched and mode locked laser the amplitude of the accumulated temperature grating depends upon the inter-pulse period $2 L_{\mathrm{c}} / c$. If the period $2 L_{\mathrm{c}} / c$ is more than $\tau$ (thermal relaxation time constant) then thermal relaxation of the medium does not effect the steady state operation of the DFDL in which it generates an the envelope of pulses. On the other hand when the inter-pulse time is less than the relaxation time, the situation becomes complex. Let the amplitude of a fringe in the temperature grating be $n_{1}$ and the accu- 
mulated amplitude $\left(n_{\mathrm{a}}\right)_{\mathrm{k}}$ where $\mathrm{k}=1,2,3 \ldots . \mathrm{n}$.

The accumulation of temperature grating in the dye medium will enhanced due to the rising intensities of the excitation pulses in the early part of the envelope. This will tend to increase the effective amplitude of a temperature grating and the subsequent intensity of the DFDL output pulses. The accumulative approach can be described as follows. The effective increase for the nth pulse will be given by. ${ }^{5}$

$$
\left(n_{\mathrm{a}}\right)_{\mathrm{n}}=\left(n_{1}\right)_{\mathrm{k}}+r\left(n_{1}\right)_{(\mathrm{k}-1)}+\cdots+r^{(\mathrm{n}-1)} n_{1}
$$

where $n_{1}$ is the amplitude of the temperature grating. The summation of terms for nth pulse may be expressed as,

$$
\left(n_{\mathrm{a}}\right)_{\mathrm{n}}=\sum_{\mathrm{k}=1}^{\mathrm{n}} n_{1(\mathrm{n}-\mathrm{k}+1)} r^{(\mathrm{k}-1)}
$$

In (6) the energy of each pulse is different from each other pulses. There are three possible cases of interest based on the interrelation of inter-pulse period $2 L_{\mathrm{c}} / c$, temperature relaxation time constant $\tau$ and pulse length $\tau_{\mathrm{p}}$. These cases are: -

$$
\begin{aligned}
& \text { Case } 1 \quad \frac{2 L_{\mathrm{c}}}{c}<\tau \text { and } \frac{2 L_{\mathrm{c}}}{c}>>\tau_{\mathrm{p}} \\
& \text { Case } 2 \quad \frac{2 L_{\mathrm{c}}}{c}<<\tau \text { or } \frac{2 L_{\mathrm{c}}}{c} \geq \tau_{\mathrm{p}} \\
& \text { Case } 3 \quad \frac{2 L_{\mathrm{c}}}{c} \geq \tau_{\mathrm{p}}, \tau
\end{aligned}
$$

$$
\text { Case } 1 \frac{2 L_{\mathrm{c}}}{c}<\tau \text { and } \frac{2 L_{\mathrm{c}}}{c}>>\tau_{\mathrm{p}}
$$

The discrete peak values of the amplitudes of the temperature gratings in a Q-switched and mode locked laser can be expressed by a Gaussian distribution as follows

$$
\left(n_{\mathrm{o}}\right)_{\mathrm{n}}=\frac{n_{\mathrm{op}}}{\sqrt{2 \pi}} \sum_{\mathrm{k}=1}^{\mathrm{n}} e^{-\left[\left(t_{\mathrm{k}}-t_{\mathrm{op}}\right)^{2} / t a\right]}
$$

where ta is a constant, $\left.t_{\mathrm{op}}=\left(t_{\mathrm{e}} / 2\right)+\left(\tau_{\mathrm{p}} / 2\right)\right), t_{\mathrm{e}}=\left(n 2 L_{\mathrm{c}} / c+n \tau_{\mathrm{p}}\right)$ and $n_{\text {op }}$ is the peak amplitude of the Q-switching envelope. The total accumulated amplitude can be written as

$$
\left(n_{\mathrm{a}}\right)_{\mathrm{n}}=\frac{\left(n_{\mathrm{o}}\right)_{\mathrm{k}} r^{(\mathrm{k}-1)}}{\sqrt{2 \pi}}\left(\sum_{\mathrm{k}=1}^{\mathrm{n}} e^{-\left[\left(t_{\mathrm{k}}-t_{\mathrm{op}}\right)^{2} / t a\right]}\right)_{z_{1}}^{z_{2}} e^{-\left[\left(z-z_{\mathrm{op}}\right)^{2} / z_{\mathrm{b}}\right]} d z
$$

where $z_{\mathrm{b}}$ is a constant, $\left.t_{\mathrm{k}}=(n-1) 2 L_{\mathrm{d}} / c+n \tau_{\mathrm{p}}\right)$ varies from 0 to the envelope length which is usually from 50 to $200 \mathrm{~ns}$. The generalized equation in terms of pulse and the envelope profile shapes can be express as

$$
\left(n_{\mathrm{a}}\right)_{\mathrm{n}}=r^{(\mathrm{k}-1)}\left(n_{\mathrm{o}}\right)_{\mathrm{k}} \sum_{\mathrm{k}=1}^{\mathrm{n}}(E P) \int_{\mathrm{z}_{1}}^{\mathrm{z}_{2}}(T F P) d z
$$

$E P=$ Envelope profile and $T F P=$ Temp fringe profile

Case $2 \frac{2 L_{\mathrm{c}}}{c}<\tau$ or $\frac{2 L_{\mathrm{c}}}{c} \geq \tau_{\mathrm{p}}$

The sequence progression in the accumulated energy is neither a geometric progression nor an arithmetic progression. Ther- mal diffusion does not occur significantly before next pulse comes in. The pulse length is small and there is no conduction during the pulse length. Let us suppose that the timing is fixed so that each next pulse adds a constant amount of energy to the dye pool. To investigate the effect of thermal heat accumulation causing population inversion we just need to consider pulses up to the time when the pulse of highest energy comes; the remaining pulses may cause further delay when $\tau$ is extremely high which is hardly to be of any truth because it then may accumulate nearly $80-90 \%$ of the pulse energy as examined by computer program. Practically $\tau$ is $15-25 \mathrm{~ns}$ and typically $21 / c$ is of the order of 5 9 ns. Further it has been notice from the computer simulation that for these ranges the peak of DFDL always starts declining. So there was no use of using full limits but one can used the full length of envelope that is in this case $110 \mathrm{~ns}$. Let us ignore the remaining pulses as the first half can exhibit the maximum delay $\tau_{\mathrm{D}}$ between the peaks of the DFDL and the excitation laser envelopes. The effective energy of the nth pulse may be given by

$$
\left(n_{\mathrm{a}}\right)_{\mathrm{n}}=\frac{\left(n_{1}\right)\left(1-r^{\mathrm{n}}\right)}{(1-r)}+\frac{r\left(n_{\mathrm{o}}\right)_{\mathrm{x}}\left[1-n r^{(\mathrm{n}-1)}+(n-1) r^{(\mathrm{n}-1)}\right]}{(1-r)^{2}}
$$

Where $\left(n_{\mathrm{o}}\right)_{\mathrm{x}}$ is the fixed amount of amplitude being added by each pulse to the subsequently induced temperature fringes. This assumption was made to simplify the formulation which allows us to write the above equation which is sum of geometrical and arithmetical progression.5) The peak energy of the pulses increases gradually. Let us assume that the mode-locking and QSwitching envelope is of Gaussian shape. The peak value of the amplitude is

$$
\left(n_{\mathrm{o}}\right)_{\mathrm{x}}=\frac{\left(n_{\mathrm{op}}\right)_{\mathrm{k}}}{\sqrt{2 \pi}} \sum_{\mathrm{k}=1}^{\mathrm{n}} e^{-\left[\left(t_{\mathrm{k}}-t_{\mathrm{oe}}\right)^{2} / t a\right]} \int_{\mathrm{z}_{1}}^{\mathrm{z}_{2}} e^{-\left[\left(z-z_{\mathrm{op}}\right)^{2} / z_{\mathrm{b}}\right]} d z
$$

Where $t_{\mathrm{k}}=t_{1} \rightarrow t_{\mathrm{n}}$. Substitution of (8) into (7) leads to the following generalized expression.

$$
\begin{aligned}
& \left(n_{\mathrm{a}}\right)_{\mathrm{k}}=\frac{n_{\mathrm{o}}\left(1-r^{\mathrm{n}}\right)}{\sqrt{2 \pi}(1-r)} \int_{\mathrm{z}_{1}}^{\mathrm{z}_{2}} e^{-\left(z_{\mathrm{y}}^{2} / z_{\mathrm{b}}\right)} d z \\
& +\frac{\left(n_{\mathrm{op}}\right)_{\mathrm{k}} r\left[1-n r^{(\mathrm{n}-1)}+(n-1) r^{(\mathrm{n}-1)}\right]}{\sqrt{2 \pi}(1-r)^{2}} \sum_{\mathrm{k}=1}^{\mathrm{n}} e^{-\left(t_{\mathrm{x}}^{2} / t a\right)} \int_{\mathrm{z}_{1}}^{\mathrm{z}_{2}} e^{-\left(z_{\mathrm{y}}^{2} / z_{\mathrm{b}}\right)} d z
\end{aligned}
$$

where $t_{\mathrm{x}}=\left(t_{\mathrm{k}}-t_{\mathrm{oe}}\right)$ and $z_{\mathrm{y}}=\left(z_{1}-z_{2}\right) / 2$. The limits have been written in the above equation up to half of the envelope, however they can be extend up to the end of the envelope too.

Case $3 \frac{2 L_{\mathrm{c}}}{c} \geq \tau_{\mathrm{p}}$ and $\tau$

This comes out to be most simple case in which a dye cell is being pumped by mode locked or repetitively pulsed laser. The typical pulse lengths are 20 to $30 \mathrm{~ns}$ and thermal relaxation time of the temperature gratings is of the order of 10 to $15 \mathrm{~ns}$. The inter-pulse durations are more than $\tau_{\mathrm{p}}$ or $\tau$. This is the most general version of the accumulative model and can be applied to the Q-switched lasers. The derivations are same as for modelocked lasers. However, the value of $r \%$ would remain zero in this case. 


\section{Discussion of results}

Theoretically, the delay between the peaks of the pump and DFDL pulses depends upon the peak pump power and physical parameters of the dye solution. The results obtained for one solution may differ from other solutions despite all the other conditions remain unchanged. In this work it was found that the value of delay changes by changing the pump powers. The same effect can be realized from (4) through (9) for the amplitude of the temperature grating. The model can predict the mutual dependence of pump power and the percentage increase in amplitude of the temperature gratings. If the delay in the peak amplitude of the grating is equated with output power from the DFDL then the model shows a good correspondence between experiment and theory. The resultant delays were determined from the data by the computer program as well as being determined by visual inspection. Variations in the envelope shapes were found in different shots due to pump laser instability. The decrease in delay between peaks of the envelope of Nd:YAG and DFDL as function of energy retained $r$ are shown in Fig. 3 .

The percentage increase in amplitude of the temperature gratings for a particular experimentally delay was estimated by creating a similar delay in the computer program. The computer program can create Q-switched and mode locked patterns with the option of different combinations of pulses and envelopes. It could give the delay assuming that some $r \%$ amplitude is increased or conversely $\mathrm{r} \%$ amplitude for certain particular delay. The experimentally measured delays of $14.4 \pm 0.5 \mathrm{~ns}$ for $2 L_{\mathrm{c}} / c=4.43 \mathrm{~ns}, 12.8 \pm 0.5 \mathrm{~ns}$ for $2 L_{\mathrm{c}} / c=5.93 \mathrm{~ns}$ and $10.9 \pm 0.5$ ns for $2 L_{\mathrm{c}} / c=8.31 \mathrm{~ns}$ correspond to computer calculated percentage increase of amplitudes of the temperature gratings of $74.2 \%, 67.5 \%$ and $57.6 \%$ respectively assuming Gaussian pulses and envelopes. If the exact shape of pulses and envelopes is known then the above $\mathrm{r} \%$ values are $100 \%$ correct otherwise the accuracy depends upon the nearness of the actual envelopes and pulses to those assumed in the computer program. A typical computer generated pattern of pump energy and accumulated increase in the amplitude of the temperature gratings is shown in Fig. 4. A delay of nearly $4 \mathrm{~ns}$ was introduced deliberately between the pump pulse (solid) and the amplitude of the temperature gratings (dotted) for clear identification of their relative amplitudes. The experimentally measured delay between the peaks of the envelopes was plotted against the percentage energy retained by the medium for similar delay in the computer-simulated results. The $\mathrm{r}$ and $\tau_{\mathrm{D}}$ were found to be related to each other by

$$
r=0.2498 \times 10^{0.033 \tau} \mathrm{D}
$$

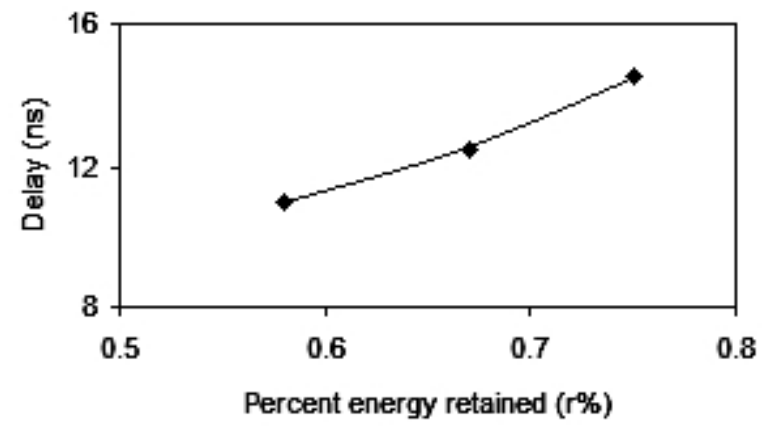

Fig. 3 Measured variation of $\tau_{\mathrm{D}}$ as a function of fraction of energy retained $r$.

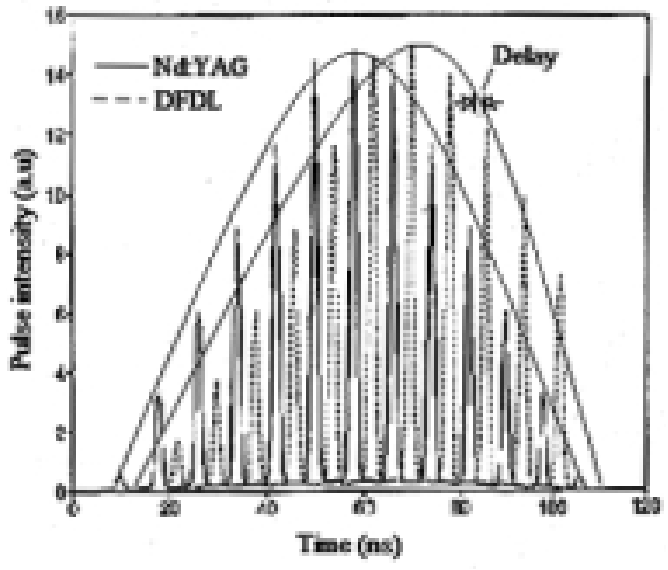

Fig. 4 Simulated delays between the peaks of the pump and the amplitudes of the refractive index grating.

The delay $\tau$ also can be expressed by ${ }^{9)}$

$$
\tau=\frac{-2 L_{\mathrm{c}} / c}{\left(\ln \left(0.2498 \times 10^{0.033 \tau}\right)\right)}
$$

The thermal relaxation time constant of $\mathrm{Rh} 6 \mathrm{G}$ in ethanol at a concentration of $1 \mathrm{mM}$ was found to be $15.8 \mathrm{~ns}$. Other researchers ${ }^{6-8)}$ have reported $\tau=12 \mathrm{~ns}$ for water and $20 \mathrm{~ns}$ for ethanol at some other concentration of Rh6G. The value of $\tau$ was also calculated using formula of ref. 8 and was found to be $16.88 \mathrm{~ns}$. Overall the relaxation times using transient grating techniques lie within 10 to $20 \mathrm{~ns}$ range. The technique is precisely applicable to determining discrete energy levels relaxation times.

\section{Conclusions}

The model for thermal heat accumulation and corresponding computer program do not include the population inversion, gain of the medium and threshold conditions. However, as assumed earlier the effective reflectivity of the thermal grating increases and results in lowering of the threshold for subsequent pulses. The rest of the relaxation processes have much shorter decay times. As the relaxation time of gain grating is very small so there is no reason to include it in the model wherein the thermal grating is under consideration which last for several nanosecond. However, it is possible to realize the evolution of an extra pulse due to increase in resultant reflectivity of the thermal grating. We have carried out calculation of increase in the amplitude of the temperature grating in a stationary dye solution. Theoretically calculated delay changes if one uses different combinations of envelope shapes and pulses. The result are sensitive to the changes in the envelope shape but are not significantly altered by changes to the pulse shape. The delay between the peaks of the Nd:YAG and DFDL pulse envelopes depends upon the peak power of the pulses, the thermal conductivity of the dye solution of the inter-pulse period of the excitation lasers. The microdensitometer records show that the DFDL pulse envelope tends to be peaked one or two inter-pulse periods after the corresponding Nd:YAG pulse envelope peak. The overall tendency of the DFDL the envelope is slowly raising front and fast decaying tail. This particular shape is formed by the gradually increasing in pump power in the first half due to rising front of the pump laser and proportionally lower increase in amplitude of grating in the second half due to decaying envelope of the 
pump laser. The thermal decay time of the gratings was found to be $15.8 \mathrm{~ns}$. The model requires some further theoretical work to get the experimentally observed results considering the gain of the medium.

\section{References}

1) N. Khan and M. Idrees: J. Biomed. Opt. 6 (2001) 1.

2) V. Yu. Kurstak and S. S. Anufrick: LFNM, 3-5 June 2002, Kharkiv, Ukraine.

3) J. Liang, H. Sun, Y. Hu, D. Sun, H.Yin, S. Wu, and Y. Liu: Optic. Commun. 216 (2003) 173.
4) A. W. Broerman, D. C. Venerus, and J. D. Schiebler: J. Chem. Phys. 11 (1999) 6955.

5) M. Fogiel: "Handbook of Mathematical Scientific and Engineering Formulas, Tables, Functions, Graphs and Transforms", TEA New York, $1986(304,7)$.

6) A. Penzkofer, W. Falkenstein, and W. Kaiser: Chem. Phys. Lett. 44 (1976) 82

7) D. Y. Key: "The scattering of light from light induced structures in liquids", Ph. D. Thesis, London University, 1977.

8) P. Y. Key and R.G. Harrison: IEEE. J. Quantum. Electron. QE-6 (1970) 645

9) N. Khan, T. A. Hall, and N. Mariun: Optics Express 11 (2003) 1520. 